\title{
tic\&société
}

Vol. 8, N 1-2 | 1er semestre 2014 et 2ème semestre 2014

Quelles perspectives critiques pour aborder les TIC ?

\section{Vers une théorie critique de l'Internet}

\section{Andrew FEENBERG}

\section{(2) OpenEdition}

\section{Journals}

Electronic version

URL: http://journals.openedition.org/ticetsociete/1382

DOI: 10.4000/ticetsociete.1382

Publisher

Association ARTIC

Electronic reference

Andrew FEENBERG, "Vers une théorie critique de l'Internet », tic\&société [Online], Vol. 8, № 1-2 | 1er semestre 2014 et 2ème semestre 2014, Online since 31 May 2014, connection on 19 April 2019. URL : http://journals.openedition.org/ticetsociete/1382 ; DOI : 10.4000/ticetsociete.1382 
tic\&société - 8 (1-2), 2014

\section{Vers une théorie critique de l'Internet}

\section{Andrew FEENBERG}

School of Communication

Chaire de recherche du Canada en philosophie de la technologie

Simon Fraser University

feenberg@sfu.ca 


\title{
Vers une théorie critique de l'Internet
}

\begin{abstract}
Andrew Feenberg est titulaire de la Chaire de recherche du Canada en philosophie de la technologie à la School of Communication de la Simon Fraser University et directeur de programme au Collège international de philosophie à Paris. II a notamment publié les ouvrages suivants: When Poetry Ruled the Streets: The May Events of 1968 (SUNY Press, 2001), Heidegger and Marcuse: The Catastrophe and Redemption of History, (Routledge, 2005), Between Reason and Experience: Essays in Technology and Modernity (MIT Press, 2010). Questioning Technology a été publié en français sous le titre [Re]penser la technique aux éditions La Découverte in 2004. Between Reason and Experience doit paraître in 2014 en français chez Lux. Son plus récent livre, The Philosophy of Praxis: Marx, Lukács and the Frankfurt School est paru aux éditions Verso en 2014.
\end{abstract}

Résumé : Le débat sur la contribution de l'Internet à la démocratie est loin d'être réglé. Certains évoquent la possibilité que l'Internet puisse restaurer la sphère publique en mentionnant le fait que les discussions en ligne favoriseraient l'empowerment. D'autres affirment que l'Internet est juste un centre commercial virtuel, l'extension finale du capitalisme global à toutes les activités de nos vies quotidiennes. Cette position plaide pour la thèse favorable à la démocratie moyennant l'intégration de quelques réserves. La contribution la plus importante de l'Internet à la démocratie ne repose pas nécessairement sur ses effets sur le processus électoral, mais plutôt sur sa capacité à rassembler une population autour de réseaux techniques qui regroupent des individus dispersés sur de vastes zones géographiques. Les patients, les joueurs de jeux vidéo, les musiciens et bien d'autres publics ont émergé sur Internet avec des conséquences surprenantes.

Mots-clés : Internet, technologie, démocratie, critique.

Abstract: The debate about the contribution of the Internet to democracy remains far from settled. Some observers point to the potential the Internet affords for restoring the public sphere, emphasizing the empowering effects of online discussions. Others claim that the Internet is little more than a virtual mall; an extension of global capitalism into every corner of our lives. Proponents of this view tend to be supportive of the democratization hypothesis, albeit with some reservations. We argue that the most important contribution of the Internet 


\section{Vers une théorie critique de l'Internet}

to democracy is not its effects on the electoral process but rather its ability to bring publics together via technical networks that link individuals dispersed over wide geographical areas. Medical patients, video game players, musical performers, and many other publics have emerged on the Internet with surprising consequences.

Keywords : Internet, technology, democracy, critique.

Resumen : El debate sobre la contribución de Internet a la democracia se encuentra lejos de estar estabilizado de manera definitiva. Unos evocan la posibilidad de que Internet restaure la esfera pública, como consecuencia del emponderamiento que suponen las discusiones en línea. Otros, afirman que Internet no es sino un centro comercial virtual, que es la extensión del capitalismo global a todas las actividades humanas. El presente artículo se sitúa en el lado de la primera opción, favorable a la democracia, si bien establece algunas reservas. La contribución más importante de Internet a la democracia no reposa necesariamente sobre los procesos electorales, sino más bien sobre su capacidad para reunir a una población en torno a redes técnicas que agrupan individuos dispersos en vastas zonas geográficas. Los pacientes, los jugadores de juegos de video, los músicos y otros públicos diversos han surgido en Internet, con consecuencias sorprendentes.

Palabras clave : Internet y democracia, esfera pública, emponderamiento 
Les technologies se stabilisent habituellement après une période où différentes configurations possibles sont en concurrence ${ }^{1}$. Dès qu'elles sont stabilisées, leurs implications politiques et sociales deviennent manifestes. Malgré une évolution sur plusieurs décennies, l'Internet demeure en transition du fait de l'apparition continuelle d'usages innovants. La nature du réseau demeure en conséquence encore non déterminée. II ne s'agit pas d'une technologie pleinement stabilisée comme le réfrigérateur ou le stylo à bille. Cela n'a cependant pas empêché la production d'une immense littérature à propos de l'internet, soit dithyrambique, soit critique de ses impacts négatifs. Certains évoquent les effets d'empowerment de l'activité en ligne lors des campagnes électorales récentes aux États-Unis et les révoltes dans le monde arabe pour faire valoir que l'Internet est un vecteur de démocratisation. D'autres prétendent que le réseau n'est qu'un centre commercial virtuel, une extension ultime de la rationalisation capitaliste dans tous les pans de notre vie, une tendance soutenue par un réseau toujours plus dense de technologies de surveillance qui menacent l'autonomie individuelle et le discours démocratique. En fait, cette controverse constitue la meilleure preuve que l'Internet n'est pas encore stabilisé. Le débat ne peut être clos alors que la controverse se poursuit avec une aussi vive intensité.

Cet article propose une approche originale pour aborder cette controverse. II reconnaît la rationalisation accrue provoquée par l'Internet tout en soulignant les formes inédites de communautés qui émergent des publics que ces technologies rassemblent. Des communautés de patients, d'amateurs de jeux vidéo, de musiciens et leurs publics, et bien d'autres groupes, sont apparus sur l'Internet et ont eu des implications surprenantes. Cet article met l'accent sur l'importance de ces communautés en tant qu'espaces de résistance. Bien que ces espaces semblent politiquement marginaux au sens usuel du terme, ils sont en fait en train de redéfinir le politique en rapport avec l'omniprésence de la technologie. Prendre en considération les relations entre l'Internet du point de vue de la rationalisation technologique d'une part et l'Internet du point de vue des initiatives sociales à caractère démocratique d'autre part permet d'avoir une perspective plus complète du réseau que si nous abordions ces deux processus séparément.

\footnotetext{
1 Cet article reprend certaines idées avancées dans l'introduction de l'ouvrage (Re)Inventing the Internet, dirigé par A. Feenberg et N. Friesen, publié en 2012 chez Sense Publishers, voir Feenberg et Friesen (2012). La traduction en français de cet article rédigé initialement en anglais a été réalisée par Oumar Kane, professeur agrégé au Département de communication sociale et publique, Université du Québec à Montréal, kane.oumar@uqam.ca
} 
Vers une théorie critique de l'Internet

La théorie critique consacrée à la technique met l'accent sur l'impact de I'Internet sur la structuration politique du monde en voie d'émerger. La technique n'est ni le domaine du consensus rationnel ni un simple outil instrumentalisé par ses propriétaires et ses gestionnaires. Les recherches menées en Science, technologie et société (STS) nous enseignent que la technologie réunit des travailleurs, des usagers, parfois même des victimes qui ont en commun le monde issu de la technique. Leur participation à ces mondes technologiques façonne la représentation qu'ils ont de leurs préoccupations et oriente leurs activités. II ne s'agit cependant pas de défendre une thèse déterministe. La technologie n'est pas une variable indépendante, elle est plutôt " co-construite " par les forces sociales qu'elle organise et mobilise. La théorie critique de la technique s'écarte de la recherche dominante en STS par la conception qu'elle se fait de ces mondes technologiques qui sont considérés comme des terrains de lutte dans lesquels des forces hégémoniques agissent et s'expriment à travers des stratégies de conception spécifiques et en opposition à des groupes subordonnés qui réussissent plus ou moins à influencer la forme future des dispositifs techniques avec lesquels ils sont en interaction. L'Internet, par le bais de la communication entre ces groupes subordonnés, entraine des conséquences sociales significatives ${ }^{2}$.

\section{L'arrière-plan historique}

La première version de ce qui est devenu l'Internet a été mise en ligne en 1969. Ce système a été baptisé ARPANET, l'acronyme de Advanced Research Projects Agency du département états-unien de la Défense qui s'était spécialisé dans les projets "blue sky ", des projets si originaux et spéculatifs qu'aucune agence gouvernementale normale n'aurait osé les financer. Il est intéressant de noter que même à un stade si précoce, plusieurs des ingénieurs impliqués dans le projet croyaient que leur travail aurait d'immenses répercussions bénéfiques. Ils ont prophétisé l'apparition d'une communauté mondiale structurée par des réseaux informatiques. Un de ces premiers enthousiastes, Vinton Cerf, a un discours aux accents poétiques lorsqu'il écrit dans son «Requiem pour l'ARPANET » :

Like distant islands sundered by the sea,

we had no sense of one community.

We lived and worked apart and rarely knew

\footnotetext{
${ }^{2}$ Pour une analyse de la relation entre la théorie médiatique et les STS, voir Boczkowski and Lievrouw (2008).
} 


\section{Andrew FEENBERG}

that others searched with us for knowledge, too...

But, could these new resources not be shared? Let links be built; machines and men be paired!

Let distance be no barrier! They set that goal: design and build the ARPANET! (cité in Abbate, $1994^{3}$ )

L'Internet s'est progressivement ouvert au public dans les années 1980 et 1990. Mais bien avant cette période, les commentateurs lui prédisaient déjà un avenir radieux en lien avec la communication médiatisée par ordinateur. En 1978, Murray Turoff et Roxanne Hiltz publient un travail d'analyse et de prospective intitulé The Network Nation (1993). Ils prévoyaient l'adoption généralisée des réseaux informatiques pour le télétravail et l'éducation. Ils croyaient que le réseau favoriserait l'égalité des sexes en raison de l'absence de présence physique. Et ils ont émis l'hypothèse que l'activité en ligne et le vote électronique redynamiseraient l'espace public dans les sociétés démocratiques. Ils ont été parmi les tout premiers à remarquer l'importance de la discussion en ligne rendue possible par des réunions asynchrones dénommées « conférences informatiques».

II est possible qu'ils aient surestimé le pouvoir de transformation de leur technologie préférée, mais leurs projections sont modérées par rapport à beaucoup d'autres qui ont suivi. Selon un tout nouveau genre de « matraquage », l'avènement de l'Internet serait un changement de la même envergure que la révolution industrielle et allait bientôt transformer chaque aspect de nos vies. Les villes seraient dépeuplées au fur et à mesure que les gens se retireraient dans des chalets électroniques isolés au fond des bois. Nos gouvernements actuels seraient remplacés par des scrutins électroniques continus. Des «agents intelligents apprendraient nos préférences et contrôleraient le monde mécanique qui nous environne sans que nous ayons à lever le petit doigt. Même le sexe serait transformé grâce à un accès à distance à des partenaires virtuels.

Bien entendu, ce battage médiatique entraina sa propre démystification. L'historien de la technologie David Noble a mis en garde de façon inquiétante contre le fait que «les visions de la démocratisation et de l'autonomisation populaire via le Net sont des illusions dangereuses; quels qu'en soient les bénéfices, ils sont massivement éclipsés et plus qu'annulés par les pertes. En

\footnotetext{
${ }^{3}$ Heureusement, Cerf est meilleur ingénieur que poète!
} 
Vers une théorie critique de l'Internet

même temps que les écrans d'ordinateur brillent d'un éclat prometteur pour une poignée d'individus, la lumière au bout du tunnel faiblit pour la multitude » (1995, p. 12). Noble exprime ainsi le scepticisme généralisé à propos de l'Internet qui est apparu dans les années 1990, au moment même où la technologie est devenue un sujet populaire de discussion. Les chercheurs critiques en sciences sociales identifient un certain nombre de phénomènes problématiques pour la démocratie. Certains prétendent que la fracture numérique exclut les pauvres tout en renforçant les acquis des plus aisés. D'autres se plaignent du fait que la discussion en ligne ne fait que renforcer les préjugés préexistants parce que, sur l'Internet, les gens n'interagissent pas avec ceux avec lesquels ils sont en désaccord. D'autres encore affirment que l'Internet est si profondément colonisé par les entreprises qu'il s'agit à peine plus qu'un véhicule publicitaire. La démocratie serait menacée par de nouvelles technologies de surveillance qui emploient le réseau pour accumuler des informations provenant de nombreuses sources, identifiant ainsi les écarts par rapport à la norme grâce au traçage et à l'extraction de données. Pourtant, même dans cette forme extrême de domination, la technologie de surveillance fait émerger des communautés temporaires de surveillés qui expriment leur dissidence indisciplinée devant la caméra. Et comme le cas Wikileaks l'a montré, la surveillance est une voie à double sens et peut parfois se retourner contre les surveillants (Yar, 2003).

Les critiques les plus acerbes de l'Internet contestent sa capacité à être au service des communautés humaines. Ils affirment que sans relation en face-àface, les gens ne peuvent pas se prendre mutuellement assez au sérieux pour former une communauté. Comment des valeurs morales peuvent-elles nous unir et des conséquences tangibles découler d'interactions qui ne sont pas plus durables qu'une fulgurance sur un écran? Comme l'a écrit Albert Borgmann: «branchés sur le réseau de communications et sur les ordinateurs, les gens semblent omniscients et omnipotents ; séparés de leur réseau, ils deviennent négligeables et désorientés. Ils ne contrôlent plus leur monde en tant que personnes à part entière. Leurs propos sont sans profondeur ni esprit, leur attention est vagabonde et vide de sens; leur sens du lieu est incertain et irrégulier »(Borgmann, 1992, p. 108) ${ }^{4}$.

En réponse à ces critiques, je soutiens que l'Internet a des implications démocratiques. Je n'exagère pas l'importance de l'Internet. C'est vrai qu'il ne remplacera pas le Parlement par une mairie électronique universelle ni ne renversera les dictatures dans le monde entier. D'un autre côté, l'exagération

\footnotetext{
${ }^{4}$ Concernant ses analyses ultérieures, voir Borgmann (2004).
} 
contraire me semble refléter un manque de perspective. Elle menace de nous faire perdre de vue les possibilités réelles qui doivent être saisies plutôt que rejetées. Ces possibilités sont en rapport avec une communauté en ligne soutenue par l'Internet, et de surcroit dédiée, comme le remarquent les critiques, à une conversation sans fin. Mais la discussion est au cœur du principe démocratique. Tout nouvel espace dans lequel la discussion émerge renforce la participation citoyenne. Dans une société de plus en plus rationalisée, où les activités des individus sont de plus en plus strictement encadrées par le monde des affaires et par le gouvernement, l'existence de cette nouvelle forme de communauté est particulièrement importante (Neyland et Woolgar, 2006).

Les critiques adressées à l'Internet sont semblables à celles concernant la télévision et, en fait, il semble que l'expérience négative de cette dernière ait influencé les appréhensions au sujet de l'Internet. Rappelons que la télévision a promis l'avènement d'un «village global » (McLuhan) dans lequel de nouvelles solidarités seraient rendues possibles par un accès aisé à l'information à propos d'autres individus et de leurs problèmes. II est vrai que des informations utiles sont diffusées dans les bulletins de nouvelles du soir, mais la propagande et la publicité ont sans doute beaucoup plus d'influence. Aldous Huxley a publié Brave New World au début des années 1930, soit quelques années seulement après les premières émissions des radios commerciales. Mais sa vision dystopique d'un public intégralement manipulé exprimait déjà cette menace très réelle. Beaucoup de chercheurs critiques en sciences sociales semblent avoir conclu que la médiation technique en tant que telle entraine l'aliénation de la masse. L'Internet s'intègre-t-il dans ce modèle explicatif ? Je ne le pense pas.

La différence entre la télévision et l'Internet est une conséquence de leurs architectures techniques respectives. En radiodiffusion, une seule source envoie des messages à une masse silencieuse. Le réseau informatique réhabilite le schéma normal de la communication humaine où les rôles d'auditeur et de locuteur sont alternés plutôt qu'exclusifs. En outre, le réseau informatique est la première forme de médiation technique réussie permettant l'activité des petits groupes. Le téléphone réunissait des paires d'interlocuteurs et la radiodiffusion s'adressait à une audience de masse. Mais jusqu'à récemment, l'immense gamme d'activités humaines qui se déroulent en petits groupes n'était pas techniquement médiatisée et ne pouvait par conséquent être réalisée qu'en face-à-face. L'Internet permet une communication interactive entre petits groupes. Les membres de ces groupes reçoivent et émettent des informations tout à la fois. II s'agit d'une avancée importante que nous avons tendance à considérer comme acquise en raison de son caractère évident après trente ans de communication en ligne généralisée. 
Vers une théorie critique de l'Internet

Les critiques sous-estiment ce phénomène et réagissent plus aux affirmations exagérées de "matraquage " relativement à l'Internet qu'à la réalité de l'expérience en ligne, y compris la leur propre. Par exemple, dans un récent article paru dans The New Yorker, Malcolm Gladwell compare défavorablement l'Internet avec les sit-in lors du mouvement des droits civiques (2010). Combien faut-il de courage pour signer une pétition en ligne, demande-til ? C'est idiot. II serait plus logique de comparer l'Internet avec les appels téléphoniques automatisés et les machines à ronéotyper utilisés pour informer les militants et imprimer des tracts au temps des sit-in plutôt que de le comparer avec les actes politiques au service desquels ces moyens de communication étaient mis.

Voici encore un autre exemple. Hubert Dreyfus concentre sa critique de I'Internet sur un groupe appelé les « Extropiens » qui sont impatients de voir le jour où leurs cerveaux pourront être téléchargés sur des ordinateurs (Dreyfus, 2001). II n'y aurait aucun intérêt à attaquer ce groupe si ce n'était pas important, mais je ne vois pas quelle importance cela peut avoir pour ceux d'entre nous qui utilisent l'Internet tous les jours tout en restant fermement immergés dans la vie matérielle. Pour compliquer encore plus les choses, à la fin de son livre, Dreyfus esquive l'accusation de technophobie en évoquant l'usage qu'il fait de l'Internet dans ses cours à Berkeley. Nulle part il ne réfléchit à la signification sociale et politique de la communauté en ligne. Au lieu de cela, il rejette la discussion en ligne comme étant triviale, car elle ne serait pas basée sur une expertise ou un engagement suffisants. Mais ce n'est là pas tant une critique de l'Internet que de la démocratie elle-même. Elle perd de vue toute idée de la grande victoire que représente la conquête de ce nouveau territoire pour la communication humaine ordinaire. II existe une longue histoire des technologies de communication pensées à l'origine pour la radiodiffusion ou les usages officiels et qui ont fini comme instruments d'interactions humaines informelles. Le téléphone, par exemple, a été initialement conçu pour les besoins du gouvernement. Lorsque les femmes se le sont approprié pour des fins sociales et familiales, les ingénieurs se sont plaints amèrement du gâchis de leur bel instrument (Fischer, 1988). Plus surprenant encore, le téléphone a d'abord été pensé comme une technologie de radiodiffusion. À l'origine, plusieurs sociétés diffusaient des spectacles musicaux en direct aux abonnés. En France, jusqu'en 1920, la société Théâtrophone diffusait des opéras par le biais du téléphone (Bertho, 1984, pp. 80-81).

Ce schéma s'est répété dans le cas des réseaux informatiques. Le premier 
réseau domestique réussi n'est pas l'Internet, mais le système Minitel français. Préoccupé par la lenteur de l'informatisation dans le pays, le gouvernement français a mis sur pied un réseau basé sur une technologie similaire à celle de I'Internet. Six millions de terminaux Minitel gratuits ont été distribués aux abonnés du téléphone au début des années 1980. Ces terminaux étaient conçus pour consulter le répertoire téléphonique électronique, les nouvelles et les petites annonces ainsi que les horaires des trains, les résultats d'examens et d'autres documents officiels du même genre. Mais peu de temps après le déploiement du système, des pirates informatiques y ont introduit la messagerie instantanée. II n'a pas fallu longtemps avant que cette application inattendue ne devienne la plus importante du Minitel. Ironiquement, la messagerie était essentiellement dédiée aux rencontres amoureuses et au sexe. Le froid nouveau support d'information a ainsi été transformé en un torride bar électronique pour célibataires (Feenberg, 2010).

Tout comme le réseau Minitel, l'Internet n'a pas été initialement conçu pour favoriser la communication humaine et il aurait pu exclure entièrement le public. Compte tenu de ses origines militaires, il aurait très bien pu en être effectivement ainsi. Mais la technologie sous-jacente de l'Internet est si puissante qu'elle pouvait difficilement être contenue. Cette technologie, appelée commutation de paquets, est utile entre autres pour la construction d'un système de communication sécurisé. C'est ce qui intéressait à l'origine l'armée. Le réseau téléphonique est vulnérable parce qu'il dépend d'un central pour connecter les interlocuteurs. Une seule bombe pouvait paralyser l'ensemble du système en détruisant le central, tandis que la commutation de paquets permet d'acheminer les messages par l'intermédiaire de nombreux ordinateurs différents. Ainsi la survie du système ne dépend pas de l'un de ses nœuds. Aussi étrange que cela puisse paraitre aujourd'hui, la communication radio entre les commandants de chars a été suggérée comme une des premières applications de la commutation de paquets. Les stratèges militaires étaient plus intéressés par la survie que par le contrôle. C'est pour cette raison que la conception était non hiérarchique et redondante, deux qualités qui plus tard se sont avérées propices à la libre circulation de l'information et à l'innovation. Ces caractéristiques de la conception originelle persistent et posent des problèmes importants aux entreprises et aux gouvernements répressifs tout en permettant tant aux activités publiques fortement engagées qu'à celles socialement stigmatisées de continuer à avoir libre cours. La conception militaire de l'Internet vient ainsi en aide aux usagers ordinaires (Abbate, 1999).

À ses débuts, ARPANET était destiné à tester la nouvelle technologie avec des chercheurs militaires affiliés aux universités. Après la Seconde Guerre mondiale, les stratèges militaires étaient convaincus que la puissance 
Vers une théorie critique de l'Internet

américaine dépendait de la recherche scientifique, et ils ont suivi les conseils des scientifiques qui leur disaient que la communication et la collaboration étaient nécessaires à la recherche. Le Pentagone espérait que les chercheurs universitaires partageraient les ressources informatiques et les données sur l'Internet. Peu de temps après que l'ARPANET fut mis en ligne, à un moment où il ne reliait que quelques universités, un ingénieur a introduit un programme de courrier électronique. À l'instar des premiers ingénieurs de la compagnie de téléphone, ceux qui étaient responsables du projet ARPANET eurent d'abord des craintes quant à une socialisation improductive sur le réseau avant d'apprécier son potentiel pour la construction de liens collectifs. C'est pourquoi ils ont permis la continuation de l'expérience du courrier électronique. Nous avons hérité des conséquences de cette décision.

Pour avoir une idée de son importance, imaginons comment nous nous sentirions si des institutions telles que les universités, les agences gouvernementales et les entreprises n'autorisaient que la communication officielle dans leurs institutions: pas de blagues, pas de remarques personnelles, aucune critique. Nous trouverions sans doute une telle censure totalitaire. L'Internet aurait pu être configuré techniquement exactement de cette manière. Le résultat aurait été le renforcement de la communication officielle pour les affaires et pour le gouvernement, sans amélioration correspondante de la communication informelle dans laquelle la vie quotidienne se déploie, y compris les conversations à visée politique qui sont le fondement de l'espace public démocratique. Cet exemple hypothétique montre la nécessité de recourir à une approche différente de celle privilégiée par ses critiques les plus sévères pour comprendre le réseau. Ceux-ci se concentrent sur la banalité de la plupart des échanges, mais ils ne parviennent pas à réaliser qu'en l'absence de possibilité de tenir un discours trivial, il ne peut y avoir de discours sérieux. Nous n'avons aucune trace des conversations tenues aux $18^{\mathrm{e}}$ et $19^{\mathrm{e}}$ siècles dans les pubs et les cafés maintenant idéalisés (peut-être à juste titre) comme lieux de naissance de l'espace public, mais il ne fait pas de doute que dans ces enceintes, beaucoup de temps a été gaspillé. Plutôt que de comparer défavorablement l'Internet avec les produits culturels édités comme les journaux, il serait plus indiqué de le comparer avec les interactions sociales qui ont lieu dans la rue. La coexistence du bien, du mauvais et du trivial y est la norme et non une insulte au bon goût ou à l'intelligence parce que nous n'avons pas d'attentes uniformes à l'égard de la rue. Dans la suite du texte, je vais présenter une approche susceptible de rendre compte autant de la présence des scories que de celle des pépites d'or dans le torrent de mots que charrie l'Internet. 
Je voudrais le faire à travers une brève évocation du rôle public de la communauté en ligne sur l'Internet. Je ne discuterai pas des innombrables exemples de politique démocratique au sens usuel du terme. La liste des activités dans lesquelles l'Internet joue un rôle s'allonge chaque année, du mouvement zapatiste mexicain aux manifestations contre la guerre en Irak qui ont eu lieu un peu partout à travers le monde en passant par les prises de position contre l'Organisation mondiale du commerce (OMC) et le Fonds monétaire international (FMI). L'Internet joue également un rôle important dans la communication électorale, l'exemple qui vient spontanément à l'esprit étant celui de la campagne de Howard Dean ou l'élection réussie de Barack Obama en 2008.

Les révoltes arabes récentes ont révélé le potentiel politique de l'Internet de manière particulièrement saisissante. La technologie y a été utilisée pour construire une opinion dissidente dans un environnement totalitaire où les médias étaient entièrement contrôlés par l'État. Durant la révolte elle-même, I'Internet a facilité la propagation du mouvement et une mobilisation rapide, modifiant ainsi les conditions spatiales et temporelles de la contestation. Dans toutes ces situations, l'Internet a brisé le quasi-monopole des réseaux officiels de presse et de télévision dominés par l'entreprise privée et par le gouvernement. II a de ce fait permis à des militants de s'organiser et de s'adresser directement à des millions d'utilisateurs de I'Internet (McCaughey et Ayers, 2003). Ces exemples me semblent fournir une preuve solide pour appuyer ma thèse, mais ils ne sont pas assez fermes pour Darin Barney, qui soutient que "ces pratiques alternatives et de résistance ne représentent jusqu'à présent qu'une goutte d'eau dans un amer océan de rencontres hégémoniques avec l'étendue de la technologie numérique et de sa culture. Pour prendre la mesure de la conjoncture actuelle, nous avons besoin de travaux rigoureux qui documentent et même favorisent les usages politiques tactiques de ces technologies, mais nous avons aussi besoin de situer ces usages dans le contexte plus large de ce qui demeure un ensemble très puissant de technologies structurées pour promouvoir et pérenniser ce que Jacques Rancière décrit comme le "pouvoir illimité de la richesse" » (Barney, 2011). Pour répondre aux objections de ce genre, il est nécessaire d'avoir un cadre théorique qui précise la substance de l'Internet politique. Après tout, comme le suggère Barney, les usages politiques pourraient être exceptionnels et l'Internet définissable par l'autoprésentation narcissique et le business. Mon principal objectif dans ce qui suit est de développer une alternative cohérente à ces évaluations critiques. En anticipant ma conclusion, qui sera détaillée cidessous, j'affirme que les usages politiques de l'Internet constituent la pointe émergée de l'iceberg, qu'ils se manifestent dans le cadre plus large d'un 
Vers une théorie critique de l'Internet

renouveau d'activité dans de nombreux types de communautés en ligne différentes et qu'ils méritent toute notre attention, et en vérité, notre soutien.

\section{Une technologie en transition}

Je voudrais commencer par quelques considérations méthodologiques essentielles. Comme je l'ai souligné au début de cet article, c'est une erreur courante que de considérer l'Internet achevé et stabilisé alors qu'il n'a pas encore réellement acquis sa structure définitive. À partir de caractéristiques qui évoluent rapidement, les critiques généralisent régulièrement dans le sens de conclusions atemporelles qui seront bientôt rendues caduques par d'autres changements. Mais comment pouvons-nous évaluer une technologie qui est encore en voie de se structurer, qui est radicalement incomplète ? Ce problème a été étudié avec des approches constructivistes dans le cadre des études en STS (Pinch et Bijker, 1987). L'idée maîtresse partagée par ces approches est négative : le succès d'une technologie ne peut pas être entièrement expliqué par ses réalisations techniques. II existe toujours des voies alternatives de développement et certaines forces sociales déterminent celles qui seront privilégiées et celles qui seront abandonnées. Derrière chacun des dispositifs techniques qui nous entourent, se trouve un halo fantomatique d'alternatives qui ont été éliminées à un certain stade et que nous avons oubliées ou qui ne subsistent que comme des illustrations pittoresques dans de vieux livres. Ce qu'il est convenu d'appeler le principe de " sous-détermination » nous enseigne que les considérations techniques seules ne peuvent pas expliquer pourquoi nous vivons avec tel rescapé particulier de ce processus d'élimination plutôt que tel autre. Ce sont les événements historiques et non la supériorité technique qui expliquent pourquoi par exemple nous utilisons des réfrigérateurs qui fonctionnent à l'électricité plutôt qu'au gaz et pourquoi nos voitures fonctionnent à l'essence plutôt qu'à l'électricité.

Pour rendre les choses encore plus compliquées, la lutte entre les alternatives ne constitue pas une concurrence directe pour atteindre le même objectif. À peu près la même technologie, avec un design légèrement différent, peut servir les intérêts et les besoins de groupes sociaux très différents. Considérons, par exemple, la différence sociale importante apportée par un changement technique aussi banal que l'introduction de rampes d'accès aux trottoirs. Dans ce cas, les droits des personnes handicapées sont incorporés dans ces rampes. Autre exemple, à ses débuts, le vélo est apparu sous deux versions principales : un type rapide avec une grande roue avant et une version plus lente, plus stable avec des roues de même taille. Aucune des deux 
versions n'était «meilleure » que l'autre. La compétition entre elles a été déterminée par le choix d'un critère qui devait ultimement prévaloir dans le monde du vélo: la vitesse ou la stabilité. Avec le recul, nous savons qui l'a emporté. Par la suite, toute l'évolution ultérieure de la bicyclette a bénéficié de ces choix initiaux réussis. L'alternative vaincue a été délaissée et s'est figée dans le temps comme un fossile de dinosaure, apparaissant aujourd'hui comme manifestement inférieure selon une illusion typique du progrès. L'ambiguïté des débuts du vélo est typique. Elle illustre ce que les constructivistes appellent la "flexibilité interprétative" des technologies. Tant que les usages de la technologie ne sont pas clairs, sa conception ne peut être normalisée. Les technologies sont plus ambiguës au début de leur développement, quand plusieurs designs sont en concurrence. Au bout du compte, l'un de ces designs l'emporte et une norme émerge. La norme s'impose généralement pour une longue période, mais elle peut être désarçonnée et la flexibilité interprétative réapparaît si les conditions qui ont favorisé la norme changent. Nous sommes entourés par ces technologies stabilisées, mais l'Internet n'en fait pas encore partie. II est encore en plein dans sa période de développement initial et est donc radicalement indéterminé.

Cette approche constructiviste considère les technologies non comme des choses, mais comme des processus dynamiques plus ou moins rapides. Le processus initialement orienté dans plusieurs directions différentes est finalement stabilisé sous une forme unique plus ou moins durable. Parce que nos vies influencées par ces formes stabilisées sont trépidantes, nous avons l'impression que les objets techniques sont des construits finalisés et stabilisés plutôt que des arrangements relativement transitoires qui peuvent entrer à nouveau en transition à une date ultérieure. Nous supposons que les fonctions qu'ils remplissent sont celles évidentes que des technologies similaires devraient remplir au lieu que de remarquer le caractère contingent de leurs fonctions par rapport à des configurations spécifiques des forces sociales qui ont à l'origine interprété les problèmes d'une façon particulière. Le constructivisme vise à surmonter cette illusion afin de rétablir une image plus précise du processus d'évolution.

Pour appliquer l'approche constructiviste à l'Internet, nous devons identifier les différentes versions du réseau qui coexistent actuellement et parmi lesquelles il faudra finalement choisir. Notons que la fermeture de l'Internet autour de l'une de ces configurations possibles n'exclut pas la survie des autres dans des rôles subordonnés. À ses débuts, la radio était dominée par une programmation éducative et publique tandis que la télévision a été pour sa part imaginée pour la surveillance et l'éducation. Ces deux technologies sont rapidement tombées sous le contrôle des milieux d'affaires et sont définies 
Vers une théorie critique de l'Internet

aujourd'hui comme des médias de divertissement. D'autres usages n'étaient pas exclus, bien que les dimensions techniques et juridiques de ces alternatives soient largement déterminées par les exigences de divertissement (McChesney, 1999). Les critiques de l'Internet pensent que la même chose s'est déjà produite dans le cas du réseau, mais ils exagèrent l'étendue du contrôle par le milieu des affaires atteint à ce jour. Un véritable réseau à vocation commerciale comme le Minitel offre des possibilités inimaginables dans le cas de l'Internet. Par exemple, le système français a été conçu pour calculer le temps passé par les utilisateurs individuels à consulter les services offerts par le système afin de les facturer à la minute par le biais de leur opérateur téléphonique. Le protocole réseau alors utilisé par France Télécom rendait cela possible tout en compliquant l'internationalisation du système. Le protocole Internet n'est pas capable de facturer de manière fiable les utilisateurs pour les services, d'où l'importance de la publicité. Mais il a d'autres caractéristiques qui lui ont permis de se propager dans le monde entier. Le business est un participant tardif et et il a encore du mal à imposer son hégémonie dans le système.

\section{Trois modèles}

J'ai soutenu que l'Internet était encore en transition, qu'il oscillait entre des possibilités alternatives de développement. J'identifie trois voies possibles pour le réseau, voies que j'appelle des «modèles" car ils aspirent à définir les caractéristiques dominantes de la technologie. Chacun de ces modèles représente une configuration possible qui pourrait prévaloir dans le futur. $\mathrm{Ce}$ sont : le modèle de l'information, le modèle de la consommation et le modèle communautaire. Comme nous allons le voir, seul le modèle communautaire est un vecteur de démocratisation de l'Internet.

\section{Le modèle de l'information}

Ce modèle a présidé aux origines de l'Internet et des systèmes similaires comme le réseau Minitel en France. II vise à améliorer la diffusion de l'information, une fonction que l'Internet remplit et continuera sans aucun doute à remplir tant qu'il existera. En tant que projet social, le réseau informatique était destiné à matérialiser les théories sociologiques de l'ère de l'information selon lesquelles la connaissance a remplacé l'industrie en tant que source principale de richesse et de pouvoir. Le modèle de l'information réalise cette vision en offrant un large accès à l'information. C'est ce qui a inspiré les tentatives pour étendre le modèle de l'information de l'univers professionnel au foyer familial dans les années 1980 en France et, une décennie plus tard, sur l'Internet. En fait, il est rapidement devenu évident que la communication personnelle était 
beaucoup plus attrayante pour les usagers de ces systèmes que tout échange d'informations économiquement important. C'est pourquoi le modèle de l'information a peu de chance de l'emporter comme modalité d'interprétation générale de la signification de l'Internet.

\section{Le modèle de la consommation}

C'est un fait curieux et assez peu connu qu'à ses débuts, l'Internet était violemment hostile au monde des affaires. Les tentatives de vendre des biens et des services par le biais du système ont été sévèrement réprimées. Toute personne qui aurait entrepris une activité commerciale aurait scandalisé la communauté et aurait été attaquée par des centaines, voire des milliers de courriels hostiles. Mais une fois que la décision a été prise au début des années 1990 de permettre l'activité commerciale sur l'Internet, un raz de marée d'initiatives corporatives a balayé l'espace virtuel plutôt calme occupé jusqu'alors par des amateurs individuels et autres professeurs d'université. L'Internet était la technologie derrière la célèbre bulle boursière dotcom/.com dont l'éclatement ultérieur n'aura pas ralenti pour longtemps le rythme de l'activité commerciale du cyberespace. Aujourd'hui, l'activité économique liée aux marchés sur l'Internet est un élément important de la prospérité des nations. Ce nouveau type de marché relie à peu de frais les personnes et les biens sur un territoire global. Les entreprises les plus rentables sur l'Internet ressemblent à eBay, elles n'ont que peu ou pas de stocks, mais elles rendent possible une connexion harmonieuse entre l'offre et la demande. Bien que le courriel demeure la fonction la plus utilisée de l'Internet, l'e-business n'est pas loin derrière.

Le modèle de la consommation a un énorme potentiel de croissance parce que le film et la télévision n'ont pas encore été entièrement adaptés au format de l'Internet. Nous pouvons nous attendre à un énorme essor dans les usages de consommation où chaque genre de divertissement enregistré devient facilement accessible. Cette perspective figure d'ores et déjà parmi les priorités réglementaires du gouvernement des États-Unis. Les entreprises de divertissement et les fournisseurs de services Internet sont pressés d'obtenir légalement le droit de transformer l'Internet en une version améliorée de la télévision en privilégiant la livraison à grande vitesse de produits de divertissement par rapport aux autres fonctions du système. Cela signifierait la fin de la "neutralité du réseau ", la règle actuelle en vertu de laquelle tous les types de communication sont techniquement traités de manière identique. Si les entreprises l'emportent, I'Internet pourrait bientôt avoir beaucoup moins d'usages désintéressés et citoyens dans la mesure où la bande passante serait monopolisée par les entreprises. Bien que jusqu'à présent ce débat soit principalement états-unien, ses effets se feraient sentir dans le monde entier, 
Vers une théorie critique de l'Internet

comme cela a été le cas avec le Digital Millennium Copyright Act. Les développements ultérieurs de la technologie seront sans doute déterminés par le cours des choses aux États-Unis dans les années à venir. Le triomphe du modèle de la consommation transformerait à la fois l'interprétation dominante du système et sa technologie.

\section{Le modèle communautaire}

L'Internet tel que nous le connaissons aujourd'hui n'est pas dominé par le monde des affaires même si ce dernier y joue un rôle majeur. Au même moment, les usagers communiquent librement dans le cyberespace, contrairement aux médias de masse tels que la télévision qui sont complètement contrôlés par les entreprises. Les deux principaux types de communication personnelle sur l'Internet sont le courriel individuel et diverses formes de communication de groupe comme les listes de diffusion, les conférences en ligne et les forums web. À l'origine, ces genres étaient distincts des pages personnelles qui contenaient des renseignements personnels. Cela a changé avec la combinaison de la communication et des contenus personnels sur des sites sociaux tels que MySpace, Facebook et les blogues, désignés collectivement comme le Web $2.0^{5}$. Des communautés se construisent autour de ces espaces d'interaction sociale virtuelle.

Le terme de "communauté " est diversement interprété, d'où l'importance de le définir dès à présent pour mon propos. Je n'utiliserai pas le terme au sens large et atténué dans lequel, par exemple, les immigrants hispanophones de Los Angeles sont appelés la « communauté hispanique ». Je n'ai pas davantage l'intention d'évoquer le contraste sociologique classique entre "communauté " et "société ». L'expression " communauté en ligne » a été initialement introduite au début des années 1980 pour décrire les utilisateurs de serveurs comme le Well ainsi que des conférences à distance sur divers ordinateurs hôtes accessibles au public. Ce qui permettait de considérer ces nouvelles formes de sociabilité comme des communautés était lié à l'existence d'interactions permanentes en petits groupes. Les premiers participants ont noté que des liens relativement forts pouvaient résulter de l'interaction à long terme autour de préoccupations ou d'intérêts partagés. L'utilisation du mot " communauté » pour décrire ces liens relève d'un usage quotidien et banal du terme. Pris en ce sens, la communauté est le lieu premier de la communication

\footnotetext{
${ }^{5}$ II vaut la peine de relever que le Web 2.0, à supposer qu'il existe, n'est pas à l'origine de l'irruption de la communauté sur l'Internet. II s'agit davantage de la concrétisation et de la combinaison de ressources communicationnelles déjà présentes dans des programmes distincts de l'environnement en ligne.
} 
humaine et du développement personnel. C'est dans ce contexte que les gens évaluent le monde autour d'eux et discutent de leurs évaluations avec les autres. Toute technologie qui offre de nouvelles possibilités pour la formation de la communauté est donc démocratiquement importante.

L'essence du modèle communautaire est la possibilité de réciprocité. Chaque participant est, au moins potentiellement, à la fois lecteur ou spectateur et producteur de contenus. Les habitudes comportementales qui prévalent dans les interactions en face-à-face, qu'elles soient positives ou négatives, sont reproduites en ligne, contrairement aux espoirs utopiques des premiers enthousiastes. Dans la plupart des cas, quelques membres actifs fournissent l'essentiel du contenu tandis que les autres agissent d'abord comme lecteurs, mais la possibilité de contribuer signifie que les participants ne sont pas de simples spectateurs. Et il existe bien entendu des groupes au sein desquels un niveau élevé de participation est atteint. Le type de logiciel a un impact sur le degré de proximité dont jouissent les membres de communautés en ligne. Les espaces ouverts à tout participant anonyme sont très différents des espaces fermés dans lesquels les personnes sont identifiées par leur nom réel. Les premiers ne sont pas des communautés dans le sens où le terme est utilisé ici. Mais les communautés en ligne sont-elles de véritables communautés qui obtiennent un engagement effectif de leurs membres? Le témoignage des participants ainsi que des recherches approfondies confirment que l'Internet est le théâtre de nouvelles formes de sociabilité qui ressemblent fortement à la communauté en face-à-face. Par exemple, des enquêtes menées dans plusieurs pays par des chercheurs japonais montrent que les principes éthiques des internautes ressemblent à leurs comportements éthiques dans la vie de tous les jours (Nara et Iseda, 2004). Ce n'est pas la technologie mais la personnalité qui détermine le comportement en ligne. Et la personnalité est précisément ce que la communauté exige, c'est-à-dire la capacité à s'engager au sein d'un collectif humain. Les comportements et les symboles qui nourrissent et soutiennent l'unité imaginée de la communauté sont régulièrement reproduits sur l'Internet. Je ne peux pas pousser ce raisonnement plus loin ici, mais il y aurait beaucoup à dire pour défendre la notion de communauté en ligne (Feenberg et Bakardjieva, 2004).

L'essence du modèle communautaire est la réciprocité. Chaque participant est à la fois lecteur ou spectateur et producteur de contenus. Nul doute que cette affirmation doit être nuancée par la reconnaissance des asymétries associées à l'activité en ligne : certains usagers contribuent beaucoup plus que d'autres et certains blogues ou pages Facebook sont beaucoup plus populaires que d'autres. En outre, un grand nombre, sinon la plupart des contributions, sont sans réponse. Néanmoins, il y a beaucoup de communications réciproques sur 
Vers une théorie critique de l'Internet

Internet. La possibilité technique de la communication réciproque distingue l'Internet des médias de masse traditionnels. Pour maintenir cette structure, le modèle communautaire exige la neutralité continue du réseau de sorte que les communications non professionnelles, non rentables et politiquement controversées ne soient pas marginalisées. II doit être possible d'introduire des designs innovants pour de nouvelles formes d'association sans passer par les goulots d'étranglement bureaucratiques ou commerciaux. L'implication des développeurs open source et d'autres bénévoles est essentielle et ne peut pas survivre à une mainmise commerciale du cyberespace. L'incorporation d'un régime strict de propriété intellectuelle à la technologie serait certainement incompatible avec la liberté des échanges communicationnels. Les conditions de la communauté sont à la fois sociales et techniques. Si le modèle communautaire devait s'imposer, les applications commerciales, ludiques et informationnelles trouveraient certainement un espace, mais elles ne pourraient pas imposer au système leurs exigences techniques spécifiques. En fait, jusqu'à présent le monde des affaires semble s'être adapté aux exigences de la communauté : la prise de contrôle commerciale de certains sites communautaires les transforme en plateformes pour la publicité sans nécessairement bouleverser leur contenu communicationnel.

Les relations entre ces trois modèles sont complexes. Elles sont caractérisées par des éléments de conflit ainsi que par des combinaisons inédites de caractéristiques provenant de chacun des trois modèles. Je vais résumer brièvement certains articles que j'ai coécrits sur l'éducation et les jeux vidéo en ligne et qui illustrent la complexité qui résulte de leur interaction. L'éducation en ligne a été inventée au début des années 1980. À l'époque, seule la discussion en ligne était possible, par conséquent la pédagogie développée était basée sur le dialogue et la collaboration. Plus tard, les administrations universitaires ont été attirées par le marché inexploité de l'apprentissage automatisé sur l'Internet. L'effondrement de ce projet a produit une situation confuse dans laquelle l'éducation en ligne signifie des choses très différentes selon les personnes. Des millions d'étudiants utilisent les sites et forums en ligne aujourd'hui. Beaucoup d'entre eux sont des apprenants adultes qui ne pourraient pas étudier dans un cadre universitaire traditionnel. Le potentiel communicationnel de l'éducation en ligne représente une importante amélioration par rapport au modèle unidirectionnel de l'apprentissage à distance traditionnel. Pour les étudiants en présentiel sur le campus, l'éducation en ligne offre des possibilités de discussion en complément aux enseignements dispensés dans les amphithéâtres traditionnels. Là encore, il s'agit d'une amélioration par rapport au cours magistral traditionnel. Néanmoins, il existe un 
risque que, parce qu'il s'agit d'une technologie nouvelle et mal connue, l'éducation en ligne fournisse un alibi pour réduire l'éducation à la délivrance mécanique de matériels didactiques. La lutte pour structurer la configuration future de l'Internet va de pair avec la controverse portant sur la meilleure façon de le mettre à profit pour l'éducation : constituer des communautés éducatives, diffuser des informations ou, plus probablement, une combinaison des deux modèles (Hamilton et Feenberg, 2012). L'industrie du jeu vidéo offre un autre exemple des interactions complexes qui caractérisent l'Internet aujourd'hui. Cette industrie est maintenant plus importante qu'Hollywood et permet à des millions d'abonnés de s'adonner aux jeux multijoueurs en ligne. Les activités ludiques des joueurs sont structurées par le code du jeu, mais les communautés en ligne les organisent dans le cadre de relations informelles qui échappent au contrôle de l'industrie. Ces communautés se forment à la fois dans et en réaction aux structures rationalisées de la technologie du jeu. Dès qu'elle est active, la communauté se bat pour reconfigurer certains aspects du jeu, mobilisant à cette intention le code et certains éléments du jeu de façon innovante et dans des contextes inédits. Des marchés nouveaux apparaissent dans lesquels les biens gagnés au jeu sont vendus aux enchères par les joueurs contre de l'argent. Des jeux sont modifiés par certains joueurs habiles en piratage. II arrive que les entreprises se plaignent de ces activités non autorisées, mais au bout du compte elles essaient habituellement plutôt de coopter ce qu'elles ne peuvent pas contrôler. L'interaction entre les concepteurs de jeux et les joueurs et entre les joueurs eux-mêmes crée un environnement aux antipodes des audiences de masse créées par la télévision (Grimes et Feenberg, 2010).

\section{L'Internet politique}

Certains commentateurs ont rapidement remarqué que les communautés en ligne se forment autour d'un intérêt ou d'une préoccupation partagée. En cela, elles diffèrent des communautés spatialisées dans lesquelles une population beaucoup plus diversifiée est reliée par un territoire. Est-ce bon ou mauvais ? Les publics désavantagés peuvent unir leurs forces en ligne et avoir ainsi un impact plus important. C'est ce qui a rendu possible pour des Américains ordinaires de rassembler d'énormes sommes d'argent pour soutenir les candidats politiques qui auraient pu être balayés des urnes par des adversaires bénéficiant du soutien de quelques riches donateurs ou d'un parti politique. D'autre part, le débat public implique le désaccord et certains affirment que le débat est affaibli à cause de l'homogénéité des groupes sur l'Internet. Pourtant, il n'est pas du tout certain que les gens s'engagent dans des échanges plus animés hors ligne que sur l'Internet. En tout cas, ceux qui s'intéressent à la politique limitent rarement l'ensemble de leurs conversations politiques à 
Vers une théorie critique de l'Internet

I'Internet. Chacun a beaucoup de contacts en face-à-face dans lesquels des désaccords peuvent surgir. Ce n'est pas une raison convaincante pour condamner l'Internet et tous ses produits. Ces débats familiers perdent de vue une question plus importante. Les implications démocratiques les plus innovantes de l'Internet commencent à peine à émerger, et elles ont moins à voir avec la politique traditionnelle qu'avec de nouvelles formes d'agency qui sont en voie de redéfinir et d'élargir la sphère du politique. Ce que nous identifions souvent comme la politique sur l'Internet est simplement un exemple de ce phénomène plus large. Pour comprendre cette nouvelle forme du politique, nous devrons encore une fois reconsidérer notre façon de penser la technique.

Jusqu'à récemment, dans les discussions sur la technique, l'accent a principalement été mis sur l'efficacité. Mais les philosophes américains de la technique soutiennent que cela est insuffisant. Langdon Winner a été parmi les premiers à soutenir que «les artefacts sont politiques » et à suggérer que la technique impose un régime quasi-constitutionnel fixant les conditions de la vie quotidienne (Winner, 1986, pp. 47 et suivantes). Dans la même veine, Lawrence Lessig affirme également que « le code est la loi » (2006). Mais si la technique a un tel pouvoir législatif, elle partage également le défaut de la plupart des législations en favorisant certains intérêts au détriment des autres. C'est pourquoi il serait souhaitable d'établir un régime technologique plus démocratique, permettant la représentation d'un large éventail d'intérêts. Mais le politique diffère de la technique à bien des égards. La représentation politique dans les républiques démocratiques a toujours été d'abord organisée autour d'unités territoriales. Les intérêts communs de ceux qui vivent ensemble fournissent une base pour des décisions communes et pour le choix des représentants. Si des désaccords surviennent, ils peuvent être surmontés par la discussion et le vote au sein de la communauté ou par une autre forme de scrutin.

La représentation est le principal moyen d'autoaffirmation de la communauté dans les démocraties modernes. C'est à travers leurs représentants que les groupes défendent leurs intérêts dans la sphère politique. Nous appelons cet exercice du pouvoir l'agency, ce qui traduit la capacité d'agir. Traditionnellement en politique, les représentants exercent l'agency au nom d'une communauté sous le contrôle de laquelle ils agissent plus ou moins. Mais les sociétés technologiques avancées rassemblent des collectifs de personnes géographiquement dispersées autour d'un certain genre de médiation technique. Les activités éducatives, le travail, les loisirs et même la maladie 
créent un monde partagé dans lequel les individus circulent tout autant que dans leur communauté locale. De nouveaux réseaux qui sont médiés par des rapports communs à la technologie émergent. Ces réseaux se superposent aux communautés géographiques et entrent en concurrence avec elles pour ce qui est de leur importance dans la vie des citoyens. Appartenir à un tel réseau revient à avoir des intérêts spécifiques qui découlent de la participation aux possibilités qu'il offre et aux problèmes qu'il engendre. J'appelle ces intérêts des " intérêts participatifs ". Ils peuvent être plus ou moins bien représentés selon le design et l'organisation du réseau, les possibilités qu'il offre à ses membres de reconnaître leur appartenance commune et le corps de connaissances qui le structure et l'organise (Feenberg, 2001).

L'Internet a le pouvoir de mettre les personnes impliquées dans ces réseaux techniquement médiés en contact les uns avec les autres. Ce qui est plus novateur et politiquement significatif au sujet de l'Internet, c'est sa capacité à faciliter une réflexion collective portant sur les intérêts des participants. Maria Bakardjieva (2009) explique l'émergence de nouvelles formes de communautés parmi les utilisateurs de l'Internet comme une réponse à un large éventail de problèmes civiques et de frustrations. Elle appelle ce phénomène le "sousactivisme ", c'est-à-dire une sorte de sphère prépolitique qui ouvre des espaces pour l'agency en relation avec des institutions telles que le système de santé, les agences gouvernementales et les écoles. Les frontières entre les univers personnel et politique, entre le «microcosme » de la vie quotidienne et la société globale se déplacent. Cela a des implications importantes pour la structure de l'espace public. En même temps que la discussion et les interventions se répandent partout où les gens identifient des intérêts communs, la vie publique embrasse des contextes formellement conçus comme relevant strictement du privé.

En 1995, j'ai étudié un exemple précoce de sous-activisme dans le domaine de la médecine. Avant même que l'Internet soit ouvert au public, des patients et leurs familles communiquaient sur les réseaux informatiques privés tels que le réseau Prodigy sur lequel j'ai trouvé un forum de discussion pour les patients atteints de la maladie neurologique ALS (dite maladie de Lou Gehrig), une affection rare. Les patients échangeaient mutuellement du soutien, discutaient de leur vie avec la maladie et partageaient des informations sur les expérimentations médicales. Ce nouveau type d'organisation des malades a bouleversé les stéréotypes portés sur ceux-ci. Au lieu d'attendre dans l'isolement une aide individualisée du corps médical, les patients se sont collectivement organisés pour promouvoir leurs intérêts en tant que patients. Ils ont finalement exercé une pression sur la ALS Society et ont exigé des Instituts nationaux de santé un budget plus important. L'ALS Society était une agence de 
Vers une théorie critique de l'Internet

santé bénévole qui défendait avant la création du forum ALS leurs intérêts face à la communauté médicale sans méthode systématique de consultation. Aujourd'hui, des forums similaires regroupant des patients foisonnent sur I'Internet et ont engendré un environnement social très différent pour la médecine (Feenberg et al., 1996). Comme le montre cet exemple, la représentation des communautés techniquement médiées est compliquée par le rôle des experts dans la création et la gestion des réseaux techniques (Feenberg, 2001). Les experts représentent la communauté constituée par un réseau technique dans le sens où ils implantent certains des intérêts participatifs de ses membres. Mais l'expertise est basée sur une connaissance technique qui, contrairement à la sagesse recherchée chez les représentants politiques, est cumulative et doit être acquise à travers une formation approfondie. Comme les technologies, les disciplines techniques sont sousdéterminées et promeuvent certains intérêts sociaux spécifiques sous des formes techniquement rationnelles. Ces corps de savoirs techniques transmis à des générations successives d'experts incorporent le résultat des luttes passées pour imposer un design particulier. Les designs actuels sont le fruit de cet héritage technique et de l'agency des participants actuels qui font pression sur ceux qui ont le contrôle de la technologie. Là où la technologie est impliquée, les coûts énormes et les importants délais dans la production d'un corps d'experts interdisent les changements brusques et radicaux. Quand de nouveaux groupes émergent, ils doivent faire partager leurs préoccupations à un corps d'experts, les convaincre de modifier les designs existants et, finalement, intégrer leurs préoccupations dans la formation de la prochaine génération d'experts. C'est tout à fait différent de la politique au sens habituel du terme. Les politiciens sont facilement remplaçables, mais pas les experts qui ont une formation technique poussée et qui doivent traduire le projet des gens dans un design technique. Dans ce dernier cas, la représentation ne change pas le personnel mais plutôt les règles selon lesquelles le personnel prend des décisions. Les intérêts participatifs des membres des communautés médiées techniquement sont donc représentés différemment des intérêts politiques des communautés à base géographique.

Considérant que les dirigeants politiques sont tenus de rendre des comptes à leurs électeurs, il serait absurde de les remplacer par un corps élu d'experts techniques susceptibles d'être révoqués. Les experts sont les détenteurs irremplaçables de traditions de savoirs qui jouent un rôle essentiel dans la vie moderne. Ils peuvent être sollicités pour exercer leur pouvoir de manière responsable, par exemple en prenant en compte les effets imprévus de leurs actions, mais cela est tout à fait différent de la reddition de comptes lors des 
scrutins comme Albena Azmanova ${ }^{6}$ l'explique. C'est ce qui motive un nombre important de programmeurs informatiques. Les utilisateurs de logiciels forment une communauté invisible qui, jusqu'à récemment, était impuissante face aux firmes géantes telles que Microsoft. La volonté de ces entreprises de répondre aux exigences des utilisateurs est très limitée. L'industrie du logiciel est jeune, et à la naissance de l'ordinateur central IBM, le logiciel a été développé par des utilisateurs plutôt que par des entreprises. Les habitudes de liberté des échanges acquises alors ont fusionné progressivement avec un mouvement idéologique pour le logiciel libre et ouvert initié par Richard Stallman en 1985, mouvement dont le développement rapide a par la suite eu un impact énorme sur l'Internet. Chaque projet logiciel rassemble une communauté en ligne qui teste les programmes et suggère ou implémente des améliorations du code. Les utilisateurs et les producteurs de logiciels ne sont plus séparés par la barrière de l'entreprise commerciale, mais en tant que lecteurs et rédacteurs dans d'autres types de forums en ligne, ils peuvent changer de rôle et interagir mutuellement. Kate Milberry analyse un cas particulièrement intéressant qui relève de ces interactions qu'elle qualifie de «techno-activisme". L'apparition d'une génération d'experts progressistes autodidactes spécialistes de la technologie de l'Internet ouvre de nouvelles possibilités. Milberry examine comment et pourquoi ces militants techno-activistes se sont approprié la technologie Wiki, l'utilisant comme un espace et un outil de communication démocratique dans le cyberespace. En retour, cela a favorisé l'émergence de nouvelles pratiques communicationnelles hors ligne, établissant ainsi une relation dialectique entre les experts techniques et le monde social qu'ils servent. La pratique démocratique en ligne préfigure une société plus juste dans laquelle les interventions démocratiques dans le développement et l'utilisation de la technologie sont consciemment organisées (Milberry, 2007).

Ces exemples montrent comment les communautés en ligne ont commencé à utiliser l'Internet pour coordonner leurs exigences pour une meilleure prise en compte des intérêts participatifs. Malgré une évolution décourageante dans d'autres domaines, dans la sphère technique l'agency est en croissance. Des militants en tous genres ont su exploiter le potentiel de l'Internet pour favoriser la discussion et l'intervention. La capacité d'agir - une manière brève de définir l'agency - a été renforcée par la disponibilité de nouveaux moyens techniques qui, bien que n'étant pas originellement destinés à ces usages, ont été adaptés pour ces fins nouvelles. Ces nouvelles formes de politique en ligne ne peuvent pas remplacer les modes de représentation traditionnelle, mais leur existence signifie que l'activité dans l'espace public peut maintenant s'élargir pour intégrer

\footnotetext{
${ }^{6}$ Azmanova développe cette distinction dans le cadre d'un travail en cours.
} 
Vers une théorie critique de l'Internet

des questions techniques auparavant considérées comme neutres et laissées sans aucune consultation à la discrétion des experts. Cela crée un environnement social et technique dans lequel l'agency dans les domaines politiques traditionnels a également commencé à se libérer de la passivité induite par des décennies d'un régime régulier de radiodiffusion.

Les défis pour la recherche présentés par cette nouvelle situation sont énormes. Les affaires politiques ne sont plus l'affaire exclusive de groupes politiques traditionnellement constitués qui débattent de questions traditionnelles. L'éventail des questions et des groupes s'élargit sans cesse de la façon la plus imprévisible qui soit. De nouveaux groupes émergent à travers des luttes destinées à créer une identité parallèlement au travail de redéfinition et de réinvention du «monde " dans lequel ils vivent (Callon et al., 2001). Les chercheurs qui se consacrent à l'étude de l'Internet doivent être attentifs à des phénomènes similaires dans l'environnement technique médié qu'ils étudient. Les exemples décrits dans cet article suggèrent un changement important dans notre environnement. La crédibilité des arguments technocratiques dans une société technologiquement avancée et le déclin corrélatif de l'agency des citoyens sont finalement remis en cause. Le retour de l'agency peut sembler apolitique, mais alors qu'est la démocratie sinon l'activité des individus pour la détermination de leur propre existence collective ? Et dans la mesure où une grande partie de la vie est maintenant médiée par la technologie, des pans de plus importants de l'existence sont susceptibles de dépendre de nouvelles formes de contrôle communautaire. Mais cela à la condition que le modèle communautaire soit capable de perdurer. C'est le défi ultime pour la communauté en ligne : préserver les conditions de la communauté sur l'Internet. Un Internet démocratique ? Cela dépend de la capacité des usagers ordinaires à défendre son potentiel démocratique dans les années à venir ».

\section{Références bibliographiques}

ABBATE J., 1994, From Arpanet to Internet: A History of ARPA-sponsored Computer Networks, 1966-1988, thèse de doctorat. University of Pennsylvania.

ABBATE J., 1999, Inventing the Internet. Cambridge (Mass.), MIT Press.

BAKARDJIEVA M., 2009, "Subactivism : Lifeworld and Politics in the Age of the Internet ", The Information Society, vol. 25, n², pp.91-104, http://dx.doi.org/10.1080/01972240802701627, dernière consultation le 26/05/2014. 
BERTHO C. 1984, Histoire des télécommunications en France, Toulouse, Erès.

BOCZKOWSKI P. et L. LIEVROUW, 2008, «Bridging STS and communication studies: Scholarship on media and information technologies", dans E. HACKETT, O. AMSTERDAMSKA, M. LYNCH et J. WACJMAN (dir.), Handbook of Science and Technology Studies, pp. 949-977. Cambridge (Mass.), MIT Press, http://polaris.gseis.ucla.edu/llievrou/BoczkowskiLievrouw.pdf, dernière consultation le 26/05/2014.

BORGMANN A., 1992, Crossing the Postmodern Divide, Chicago, University of Chicago Press.

BORGMANN A., 2004, "Is the Internet the Solution to the Problem of Community ", dans A. FEENBERG et D. BARNEY, Community in the Digital Age, Lanham (MD), Rowman and Littlefield, pp. 53-67.

CALLON M., P. LASCOUMBES et Y. BARTHE, 2001, Agir dans un Monde Incertain, Paris, Le Seuil.

DREYFUS H., 2001, On the Internet, Londres, Routledge.

FEENBERG A., 2001, (Re)penser la Technique, Paris, La Decouverte, http://www.journaldumauss.net/spip.php?article817, consulté le 26/05/2014.

FEENBERG A., 2010, Between Reason and Experience. Cambridge (Mass.), MIT Press.

FEENBERG A. et M. BAKARDJIEVA, 2004, "Consumers or Citizens? The Online Community Debate », dans A. FEENBERG et D. BARNEY (dir.), Community in the Digital Age, Lanham (MD), Rowman and Littlefield, pp. 1-26.

FEENBERG A. et N. FRIESEN (dir.), 2012, (Re)Inventing the Internet, Rotterdam, Sense Publishers.

FISCHER C., 1988, "Touch Someone": The Telephone Industry Discovers Sociability ", Technology and Culture 29, pp. 32-61.

GRIMES S. et A. FEENBERG, 2010, "La rationalisation du jeu: Une théorie critique du jeu numérique ", Appareil : Esthetique \& Société, https://www.sfu.ca/ andrewt/books/La\%20Rationalisation\%20du\%20jeu.pdf, dernière consultation le 26/05/2014.

HAMILTON T. et A. FEENBERG, 2012, "Alternative Rationalisations and Ambivalent Futures: A Critical History of Online Education", dans A. FEENBERG et N. FRIESEN (dir.), (Re)Inventing the Internet, Rotterdam, Sense Publishers, pp. 43-70.

GLADWELL M. 2010, «Why the revolution will not be tweeted », The New Yorker, 
4 Octobre, http://www.newyorker.com/reporting/2010/10/04/101004fa fact gladwell?curren $\underline{\text { tPage}=a l l}$, dernière consultation le 26/05/2014.

HILTZ S. et M. TUROFF, 1993, The Network Nation, Cambridge (Mass.), MIT Press.

LESSIG L., 2006, Code: and Other Laws of Cyberspace Version 2.0, New York, Basic Books.

McCAUGHEY M. et M. AYERS, 2003, Cyberactivism : Online Activism in Theory and Practice, New York, Routledge.

McCheSNeY R., 1999, Rich Media, Poor Democracy : Communication Politics in Dubious Times, Urbana and Chicago, University of Illinois Press.

NEYLAND D. et S. WOOLGAR, 2006, «Governance and Accountability Relations in Mundane Techno-Scientific Solutions to Public Problems ", https://www.esrc.ac.uk/.../23529da4-cbc8-403e-829d..., dernière consultation le 26/05/2014.

NOBLE D, 1995, «The Truth about the Information Highway », Monthly Review Archives, http://archive.monthlyreview.org/index.php/mr/article/view/MR-047-021995-06 6, dernière consultation le 26/05/2014.

PINCH T. et W. BIJKER, 1987, «The Social Construction of Facts and Artefacts », dans W. BIJKER, T. HUGHES et T. PINCH (dir.), The Social Construction of Technological Systems, Cambridge (Mass.), MIT Press. 